\title{
Pion transverse-momentum spectrum, elliptic flow, and Hanbury-Brown-Twiss interferometry in a viscous granular source model $^{*}$
}

\author{
Jing Yang (杨婧) ${ }^{1}$ Wei-Ning Zhang (张卫宁) $)^{1,2 ; 1)} \quad$ Yan-Yu Ren (任延宇) ${ }^{2}$ \\ ${ }^{1}$ School of Physics and Optoelectronic Technology, Dalian University of Technology, Dalian, Liaoning 116024, China \\ ${ }^{2}$ Department of Physics, Harbin Institute of Technology, Harbin, Heilongjiang 150006, China
}

\begin{abstract}
We examine the evolution of quark-gluon plasma (QGP) droplets with viscous hydrodynamics and analyze the pion transverse-momentum spectrum, elliptic flow, and Hanbury-Brown-Twiss (HBT) interferometry in a granular source model consisting of viscous QGP droplets. The shear viscosity of the QGP droplet speeds up and slows down the droplet evolution in the central and peripheral regions of the droplet, respectively. The effect of the bulk viscosity on the evolution is negligible. Although there are viscous effects on the droplet evolution, the pion momentum spectrum and elliptic flow change little for granular sources with and without viscosity. On the other hand, the influence of viscosity on HBT radius $R_{\text {out }}$ is considerable. It makes $R_{\text {out }}$ decrease in the granular source model. We determine the model parameters of granular sources using the experimental data of pion transversemomentum spectrum, elliptic flow, and HBT radii together, and investigate the effects of viscosity on the model parameters. The results indicate that the granular source model may reproduce the experimental data of pion transverse-momentum spectrum, elliptic flow, and HBT radii in heavy-ion collisions of $\mathrm{Au}-\mathrm{Au}$ at $\sqrt{s_{N N}}=200 \mathrm{GeV}$ and $\mathrm{Pb}-\mathrm{Pb}$ at $\sqrt{s_{N N}}=2.76 \mathrm{TeV}$ in different centrality intervals. The viscosity of the droplet leads to an increase in the initial droplet radius and a decrease of the source shell parameter in the granular source model.
\end{abstract}

Key words: high-energy heavy-ion collisions, pion interferometry, elliptic flow, granular source, viscous hydrodynamics

PACS: $25.75 .-\mathrm{q}, 25.75 . \mathrm{Gz}$

\section{Introduction}

At sufficiently high temperature and/or high density, quantum chromodynamics (QCD) predicts a transition from hadronic matter to the de-confined phase of quarks and gluons, called Quark-Gluon Plasma (QGP) [1]. It is the primary goal of ultrarelativistic heavy ion collisions to create the QGP and study its evolution and detection [2 5]. Relativistic hydrodynamics is an efficient tool for describing the evolution of the hot and dense matter produced in high-energy heavy-ion collisions [6 20]. Ideal hydrodynamics, with the strong assumptions that the fluid evolves without dissipation, has demonstrated that it can reproduce many experimental results for heavy-ion collisions at the Relativistic Heavy Ion Collider (RHIC) [2 $5,21,25]$. However, dissipative evolution of the hot and dense matter is a more general case, and viscous hydrodynamic models have recently been applied in heavy ion collisions at the RHIC and the Large Hadron Collider (LHC) 9 20, 26 52].
Because of the complexity of the processes of highenergy heavy-ion collisions, it is hard to obtain the final-state results directly from first-principle calculations of QCD, the fundamental theory of strong interaction. Phenomenologically, starting from the experimental data of final observables one may infer the processes backward step-by-step with models. In Refs. 53] and [54], we systematically investigated the pion transversemomentum spectrum, elliptic flow, and Hanbury-BrownTwiss (HBT) interferometry in a granular source model of QGP droplets and compared the model results of these observables with the experimental data of heavy-ion collisions at the RHIC and LHC. In this model, granular sources are assumed to be formed at a later time in the QGP expansion. The lumps of QGP after this time are dealt with as spherical droplets for simplicity, which evolve in ideal hydrodynamics as in previous granular source models $[55$ [59]. The anisotropic pressure gradient in the early QGP matter is assumed to lead to the anisotropic initial velocities of the QGP droplets in the

Received xx February 2017

* Supported by National Natural Science Foundation of China (11675034 and 11275037)

1) E-mail:wnzhang@dlut.edu.ac.cn

(c) 2013 Chinese Physical Society and the Institute of High Energy Physics of the Chinese Academy of Sciences and the Institute of Modern Physics of the Chinese Academy of Sciences and IOP Publishing Ltd 
granular source model. The investigations indicated [53] that the granular source model consistently reproduces the data of pion transverse-momentum spectrum, elliptic flow, and HBT radii in heavy ion collisions at the RHIC and the LHC. On this basis, developing a viscous granular source model and examining the effect of viscosity on the source evolution and the multi-observable analyses in the viscous granular source model are the motivations of this work. Furthermore, experimental data for different observables at different collision energies and in different centrality intervals give strict constraints on the model parameters. This makes these parameters include useful information about the source geometry and dynamics. They may provide helpful information for the study of the QGP properties and dynamics at earlier stages. Therefore, it is of interest to investigate the applicability of the parameters and their modification in a viscous granular source model.

In this paper, we use viscous relativistic hydrodynamics to describe droplet evolution, and construct the granular source with the evolving droplets. We analyze the pion transverse-momentum spectrum, elliptic flow, and HBT interferometry in the viscous granular source model. The model parameters of granular sources are determined by the experimental data of pion transversemomentum spectrum, elliptic flow, and HBT radii together. The effects of viscosity on the model parameters are investigated. We find that the motion of droplets in the granular sources reduces the effect of viscosity on the pion transverse-momentum spectrum and the viscosity causes a small decrease of the elliptic flow. However, viscosity makes the HBT radius $R_{\text {out }}$ decrease significantly in the granular source model. The model results of the granular sources are in accordance with the experimental data of $\mathrm{Au}-\mathrm{Au}$ collisions at the RHIC and $\mathrm{Pb}-\mathrm{Pb}$ collisions at the LHC. This means that the granular source model reflects in some degree the physics of the system evolution during the later stages of heavy-ion collisions.

The rest of this paper is organized as follows. In Section 2, we review the second order Müller-Israel-Stewart formalism for viscous relativistic hydrodynamics. We also present the equations of the viscous hydrodynamics in spherical geometry and provide their numerical solutions for the droplet. In Section 3, we analyze the pion transverse-momentum spectrum, elliptic flow, and HBT interferometry for viscous granular sources for heavy-ion collisions of $\mathrm{Au}-\mathrm{Au}$ at $\sqrt{s_{N N}}=200 \mathrm{GeV}$ and $\mathrm{Pb}-\mathrm{Pb}$ at $\sqrt{s_{N N}}=2.76 \mathrm{TeV}$. In Section 4, we compare the results of the observables for granular sources with different modelparameter sets and investigate the influence of viscosity on the parameters. Finally, we provide a summary and discussion in Section 5.

\section{Evolution of QGP droplets with vis- cous hydrodynamics}

In our granular source model, the source evolution is regarded as the superposition of the evolutions of the QGP droplets, and the droplets are assumed to have spherical geometry for simplicity [53 59]. In this section, we will present briefly the description of viscous hydrodynamics for spherical QGP droplets, and show their hydrodynamical evolution.

\subsection{Viscous hydrodynamics for spherical QGP droplets}

For the zero-net-baryon systems produced in ultrarelativistic heavy ion collisions, the description of viscous hydrodynamics starts from the local conservation of energy and momentum,

$$
d_{\mu} T^{\mu \nu} \equiv \partial_{\mu} T^{\mu \nu}+\Gamma_{\mu \lambda}^{\mu} T^{\lambda \nu}+T^{\mu \lambda} \Gamma_{\lambda \mu}^{\nu}=0 .
$$

Here, $d_{\mu}$ denotes the covariant differential, and the Christoffel symbol is given by

$$
\Gamma_{\alpha \beta}^{\gamma} \equiv \frac{1}{2} g^{\gamma \sigma}\left(\partial_{\alpha} g_{\beta \sigma}+\partial_{\beta} g_{\sigma \alpha}-\partial_{\sigma} g_{\alpha \beta}\right),
$$

where $g^{\mu \nu}=\operatorname{diag}(+,-,-,-)$ is a metric tensor. We adopt the Landau-Lifshitz frame [60], i.e., the local rest frame of energy density. The energy-momentum tensor $T^{\mu \nu}$ is

$$
T^{\mu \nu}=\epsilon u^{\mu} u^{\nu}-(P+\Pi) \Delta^{\mu \nu}+\pi^{\mu \nu},
$$

where $\epsilon$ is the local energy density, $u^{\mu}$ is the four-velocity of the energy flow, $P$ and $\Pi$ are the thermal equilibrium pressure and the bulk viscous pressure respectively, $\Delta^{\mu \nu} \equiv g^{\mu \nu}-u^{\mu} u^{\nu}$, and $\pi^{\mu \nu}$ is the shear viscous pressure tensor.

Based on the second-order Müller-Israel-Stewart theory of dissipative hydrodynamics [61], $\pi^{\mu \nu}$ and $\Pi$ satisfy the viscous relaxation equations

$$
\begin{aligned}
\tau_{\pi} \Delta^{\mu \alpha} \Delta^{\nu \beta} D \pi_{\alpha \beta}+\pi^{\mu \nu} & \\
& =2 \tilde{\eta} \nabla^{<\mu} u^{\nu>}-\frac{1}{2} \pi^{\mu \nu} \tilde{\eta} T d_{\lambda}\left(\frac{\tau_{\pi} u^{\lambda}}{\tilde{\eta} T}\right)
\end{aligned}
$$

and

$$
\tau_{\Pi} D \Pi+\Pi=-\tilde{\xi} d_{\mu} u^{\mu}-\frac{1}{2} \Pi \tilde{\xi} T d_{\lambda}\left(\frac{\tau_{\pi} u^{\lambda}}{\tilde{\xi} T}\right),
$$

respectively. Here, $\tilde{\eta}$ is the shear viscosity coefficient, $\tilde{\xi}$ is the bulk viscosity coefficient, $\tau_{\pi}$ is the relaxation time for shear viscosity, and $\tau_{\Pi}$ is the relaxation time for bulk viscosity. In our numerical calculations, quantities $\tilde{\eta}, \tilde{\xi}$, $\tau_{\pi}$, and $\tau_{\Pi}$ are taken to have certain relationships with 
entropy density which will be discussed later. Some notations used in Eqs. (4) and (5) are defined as

$$
\begin{aligned}
& d_{\mu} u^{\nu} \equiv \partial_{\mu} u^{\nu}+\Gamma_{\alpha \mu}^{\nu} u^{\alpha}, \\
& D \equiv u^{\mu} d_{\mu}, \quad \nabla^{\mu} \equiv \Delta^{\mu \nu} d_{\nu}, \\
& \nabla^{<\mu} u^{\nu>} \equiv \nabla^{(\mu} u^{\nu)}-\frac{1}{3} \Delta^{\mu \nu} d_{\lambda} u^{\lambda}, \\
& A^{(\alpha \beta)} \equiv \frac{1}{2}\left(A^{\alpha \beta}+A^{\beta \alpha}\right) .
\end{aligned}
$$

For a spherical droplet, Eqs. (1), (4), and (5) can be written as follows (see Appendix A for detailed derivations), which are suitable for solving numerically:

$$
\left\{\begin{array}{l}
\frac{\partial}{\partial t} E+\frac{\partial}{\partial r}\left[\left(E+P_{r}\right) v_{r}\right]=-\left(E+P_{r}\right) \frac{2 v_{r}}{r}, \\
\frac{\partial}{\partial t} M+\frac{\partial}{\partial r}\left[M v_{r}+P_{r}\right]=-M \frac{2 v_{r}}{r}-\frac{2 \tau^{r r}-\tau^{\theta \theta}-\tau^{\phi \phi}}{r}, \\
\frac{\partial}{\partial t} \tau^{r r}+\frac{\partial}{\partial r}\left[v_{r} \tau^{r r}\right]=\sigma^{r r}\left(2 \frac{1}{\gamma} \frac{\tilde{\eta}}{\tau_{\pi}}\right)+\tau^{r r}\left[-\frac{1}{\gamma} \frac{1}{\tau_{\pi}}-\frac{1}{2} \frac{1}{\gamma} \Theta-\frac{1}{2} \frac{1}{\gamma} \frac{\tilde{\eta} T}{\tau_{\pi}} D\left(\frac{\tau_{\pi}}{\tilde{\eta} T}\right)+\frac{\partial v_{r}}{\partial r}\right] \\
\frac{\partial}{\partial t} \tau^{\theta \theta}+\frac{\partial}{\partial r}\left[v_{r} \tau^{\theta \theta}\right]=\sigma^{\theta \theta}\left(2 \frac{1}{\gamma} \frac{\tilde{\eta}}{\tau_{\pi}}\right)+\tau^{\theta \theta}\left[-\frac{1}{\gamma} \frac{1}{\tau_{\pi}}-\frac{1}{2} \frac{1}{\gamma} \Theta-\frac{1}{2} \frac{1}{\gamma} \frac{\tilde{\eta} T}{\tau_{\pi}} D\left(\frac{\tau_{\pi}}{\tilde{\eta} T}\right)+\frac{\partial v_{r}}{\partial r}\right] \\
\frac{\partial}{\partial t} \tau^{\phi \phi}+\frac{\partial}{\partial r}\left[v_{r} \tau^{\phi \phi}\right]=\sigma^{\phi \phi}\left(2 \frac{1}{\gamma} \frac{\tilde{\eta}}{\tau_{\pi}}\right)+\tau^{\phi \phi}\left[-\frac{1}{\gamma} \frac{1}{\tau_{\pi}}-\frac{1}{2} \frac{1}{\gamma} \Theta-\frac{1}{2} \frac{1}{\gamma} \frac{\tilde{\eta} T}{\tau_{\pi}} D\left(\frac{\tau_{\pi}}{\tilde{\eta} T}\right)+\frac{\partial v_{r}}{\partial r}\right] \\
\frac{\partial}{\partial t} \Pi+\frac{\partial}{\partial r}\left[v_{r} \Pi\right]=\Theta \frac{1}{\gamma} \frac{\tilde{\xi}}{\tau_{\pi}}+\Pi\left[-\frac{1}{\gamma} \frac{1}{\tau_{\pi}}-\frac{1}{2} \frac{1}{\gamma} \Theta-\frac{1}{2} \frac{1}{\gamma} \frac{\tilde{\xi} T}{\tau_{\pi}} D\left(\frac{\tau_{\pi}}{\tilde{\xi} T}\right)+\frac{\partial v_{r}}{\partial r}\right]
\end{array}\right.
$$

where

$$
\begin{gathered}
E=\gamma^{2}\left(\epsilon+v_{r}^{2} P_{r}\right), \\
P_{r}=P+\Pi+\tau^{r r}, \\
M=v_{r}\left(E+P_{r}\right),
\end{gathered}
$$

and $\tau^{r r}, \tau^{\theta \theta}$, and $\tau^{\phi \phi}$ are three quantities introduced in the nonzero components of $\pi^{\mu \nu}$ as

$$
\pi^{\mu \nu}=\left(\begin{array}{cccc}
\gamma^{2} v_{r}^{2} \tau^{r r} & \gamma^{2} v_{r} \tau^{r r} & 0 & 0 \\
\gamma^{2} v_{r} \tau^{r r} & \gamma^{2} \tau^{r r} & 0 & 0 \\
0 & 0 & r^{-2} \tau^{\theta \theta} & 0 \\
0 & 0 & 0 & (r \sin \theta)^{-2} \tau^{\phi \phi}
\end{array}\right)
$$

where

$$
\tau^{r r}+\tau^{\theta \theta}+\tau^{\phi \phi}=0
$$

from the traceless condition $\pi_{\mu}^{\mu}=0$. The other referred quantities in Eq. (7) are given by

$$
\begin{aligned}
& \Theta=\frac{\partial \gamma}{\partial t}+\frac{\partial\left(\gamma v_{r}\right)}{\partial r}+2 \gamma \frac{v_{r}}{r} \\
& \sigma^{r r}=-2 \sigma^{\theta \theta}=-2 \sigma^{\phi \phi}=2\left(\gamma \frac{v_{r}}{r}-\frac{1}{3} \Theta\right)
\end{aligned}
$$

\subsection{Hydrodynamical evolution of QGP droplets}

In equation set (7), there are eight variable quantities, $E($ or $\epsilon), P_{r}($ or $P), M\left(\right.$ or $\left.v_{r}\right), \tau^{r r}, \tau^{\theta \theta}, \tau^{\phi \phi}$,
$\Pi$, and $T$. With the traceless condition [Eq. (12)], there are seven independent variables. An equation of state (EOS), $P(\epsilon, T)$, is needed when solving the hydrodynamic equations. In the numerical calculations in this paper, we use the EOS s95p-PCE [38], which combines the lattice QCD data at high temperature and the hadron resonance gas at low temperature. The equations in (77) have a similar form, $\partial_{t} U+\partial_{r} F(U)=G(U)$. They can be solved by the HLLE algorithm [6, 55, 62 64].

The initial energy density distribution of a droplet in our granular source model is taken to be the WoodsSaxon form as in Ref. 54],

$$
\epsilon(r)=\epsilon_{0} \frac{1}{e^{\frac{r-r_{0}}{a}}+1},
$$

where $\epsilon_{0}$ is the initial energy density at the center of the droplet, $r_{0}$ denotes the initial droplet radius, and $a$ is the Woods-Saxon width parameter, which is taken to be $0.1 r_{0}$. The shear and bulk viscosity quantities, $\tau^{r r}, \tau^{\theta \theta}$, $\tau^{\phi \phi}$, and $\Pi$ are set to be zero initially because the droplet is initially at rest in the local frame.

When solving equation set (7) numerically, we also need to specify the viscosity coefficients $\tilde{\eta}$ and $\tilde{\xi}$ and the relaxation time $\tau_{\pi}$ and $\tau_{\Pi}$. For the strongly coupled QGP matter produced in ultrarelativistic heavy ion collisions, the ratio of the shear viscosity to entropy density, $\tilde{\eta} / s$, is expected to be between $0.08-0.24$ [16, 30], that is 1 to 3 times the minimum $(\tilde{\eta} / s)_{\min }=1 / 4 \pi[65]$. To look into 
the effects of viscosity on observables, we take the upper bound of $\tilde{\eta} / s$ (3 times the minimum) in calculations. The shear relaxation time $\tau_{\pi}$ is taken as $3 \tilde{\eta} /(s T)$ as in Refs. [16, 35 38]. For bulk viscosity, the minimum $(\tilde{\xi} / s)_{\min }$ obtained by the AdS/CFT result is $2(\tilde{\eta} / s)_{\min }\left(1 / 3-c_{s}^{2}\right)$ [66]. Here, $c_{s}$ is the speed of sound. The entropy density $s$ and the speed of sound $c_{s}$ as functions of energy density or temperature are given by the EOS s95p-PCE (see Fig. 2 and Eq. (A2) in Ref. 38]). We take $\tilde{\xi} / s$ also as 3 times the minimum $(\tilde{\xi} / s)_{\min }$ in calculations. The bulk relaxation time is taken to be the parametrization, $\tau_{\Pi}=\max [120 \cdot \tilde{\xi} / s, 0.1] \mathrm{fm} / c$, as in Ref. 35].

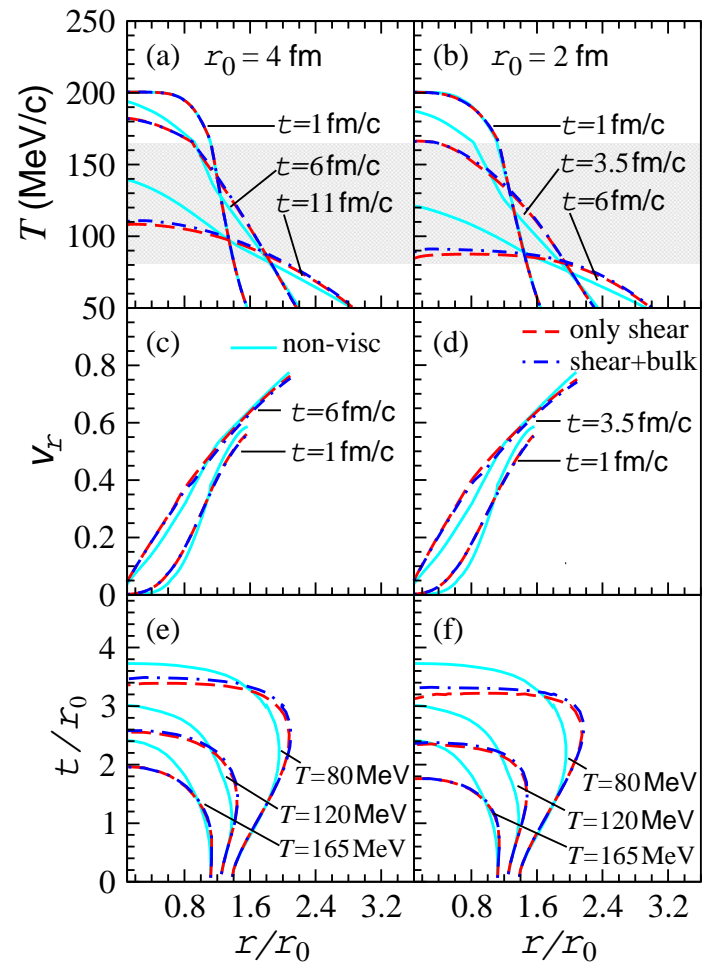

Fig. 1. (Color online) Temperature evolution [(a) and (b)], expansion velocity [(c) and (d)], and isothermals [(e) and (f)] of droplets with different initial radii and initial energy density $\epsilon_{0}=2.2$ $\mathrm{GeV} / \mathrm{fm}^{3}$.

We plot in Fig. 1 the temperature evolution (top panels), expanding velocity (middle panels), and isothermals (bottom panels) of the droplet with initial radii $r_{0}=4$ and $2 \mathrm{fm}$. Here the cyan solid lines, red dashed lines, and blue dot-dashed lines are for droplets without viscosity, with only shear viscosity, and with both shear and bulk viscosities. The initial energy density is taken as $\epsilon_{0}=2.2$ $\mathrm{GeV} / \mathrm{fm}^{3}$ [53, 54], corresponding to the moment of granular source formation. Also, the marked time in Fig. 1 is evolution time relative to the formation time. The shear viscosity of the droplet has a dual effect on the droplet evolution in the edge and central regions of the droplet.
In the edge region, the large pressure gradient leads to a fast expansion and the viscosity plays to resist the expansion. So, the temperature of a viscous droplet decreases a little slower than that of a droplet without viscosity. However, the expansion velocity is small in the central region of the droplet because of small pressure gradient. In this case, the viscosity pulls the matter in the central region to move and leads to a lower temperature of the viscous droplet compared to that of the droplet without viscosity in the central region. The dual effects on the droplet expansion velocity and isothermals can also be observed in the middle and bottom panels of Fig. 1. By comparing the curves, considering only shear viscosity and both shear and bulk viscosities, one can see that the influence of bulk viscosity on the evolution is very small.

In our granular source model, the particles are emitted from the evolving droplets. To investigate the pion transverse-momentum $p_{T}$ spectrum of the granular source, we first examine the $p_{T}$ spectrum of pions emitted from a single droplet. The pion transverse-momentum spectrum for the evolving single droplet are calculated with the Cooper-Frye formula [67]

$$
\frac{1}{2 \pi} \frac{d^{2} N}{p_{T} d p_{T} d y} \propto \frac{1}{(2 \pi)^{3}} \int_{\Sigma} p^{\mu} d \sigma_{\mu}\left(f_{0}+\delta f\right),
$$

where $y$ is particle rapidity, $f_{0}$ is the Bose-Einstein distribution, and $\delta f$ is given by [12, 29, 38],

$$
\delta f=f_{0}\left(1+f_{0}\right) \frac{p^{\mu} p^{\nu}}{2(\epsilon+P) T^{2}}\left(\pi_{\mu \nu}-\frac{2}{5} \Pi \Delta_{\mu \nu}\right) .
$$

The integration in Eq. (16) is over the hypersurface at freeze-out temperature $T_{f}$.

In Fig. 2, we plot the transverse-momentum spectra of pions emitted from droplets evolving as shown in Fig. 1 in the freeze-out temperature region $80<T_{f}<T_{c}$ $\left(T_{c}=165 \mathrm{MeV}\right)$, with the following probability used in the granular source models [53, 54, 57 59],

$$
\frac{d P}{d T_{f}} \propto f_{\text {dir }} e^{-\frac{T_{c}-T_{f}}{\Delta T_{\operatorname{dir}}}}+\left(1-f_{\mathrm{dir}}\right) e^{-\frac{T_{c}-T_{f}}{\Delta T_{\mathrm{dec}}}} .
$$

Here, $f_{\text {dir }}$ is the fraction of direct emission around $T_{c}$, and $\Delta T_{\text {dir }}$ and $\Delta T_{\text {dec }}$ are the temperature widths for the direct and decay emissions, respectively. In the calculations, we take $f_{\text {dir }}=0.75, \Delta T_{\text {dir }}=10 \mathrm{MeV}$, and $\Delta T_{\mathrm{dec}}=90 \mathrm{MeV}$ as in [53, 54]. In the edge region of the droplet, the large expansion velocity causes the large average transverse momentum of the particles. The viscous droplet in the edge region evolves more slowly (thus has higher temperature) than the droplet without viscosity. So, the transverse momentum spectrum for viscous droplets is higher at large $p_{T}$ than that for droplets without viscosity. This effect is more obvious for smaller droplets. The pressure gradient in smaller droplets is larger than in larger droplets with the same initial energy density. 


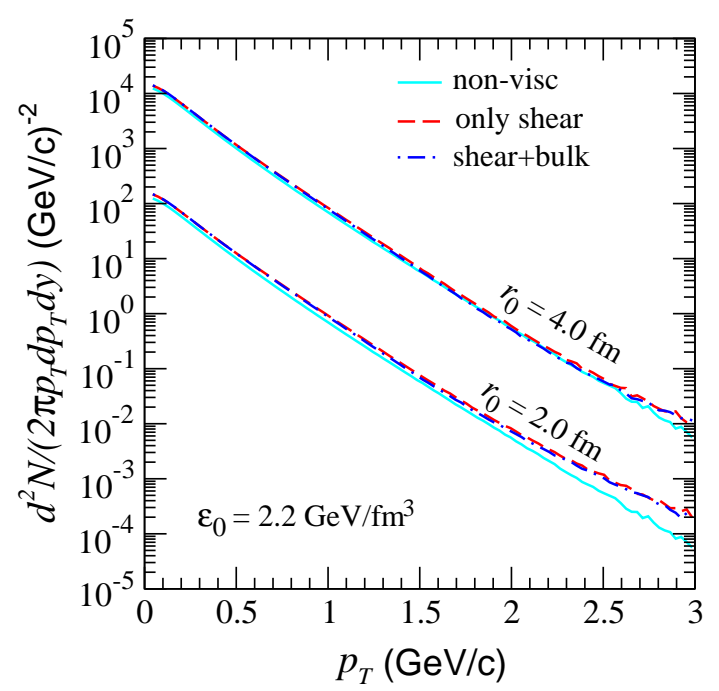

Fig. 2. (Color online) Transverse-momentum spectra of pions emitted from evolving droplets as in Fig. 1 and with the freeze-out temperature distribution (18).

\section{Multi-observable analyses for granular sources}

Single-particle momentum spectra, elliptic flow, and two-particle HBT correlations are important observables in high-energy heavy-ion collisions. They are tightly related to the initial conditions, evolution, and freeze-out of the particle-emitting sources. In this section, we will analyze these observables for the granular sources for heavy ion collisions at RHIC and LHC energies, and investigate the effects of viscosity on the analysis results.

\subsection{Ingredients of granular source model}

In Refs. 55 57, a granular source model of QGP droplets was proposed and developed by Wei-Ning Zhang et al. to explain the RHIC HBT puzzle, $R_{\text {out }} / R_{\text {side }} \sim 1$ 68 71], where $R_{\text {out }}$ and $R_{\text {side }}$ are two HBT radii in the transverse plane along and perpendicular to the transverse momentum of the particle pairs [72, 73]. Although the early idea of constructing the granular source model was based on the first-order QCD transition [55, 74 77], the occurrence of granular sources may not be limited to the first-order phase transition. There are other factors, such as large initial fluctuations and some system instabilities may lead to the granular inhomogeneous structure of particle-emitting sources in ultrarelativistic heavy-ion collisions [56 59, 64, 78, 84]. In the granular source model, the droplets are assumed to have spherical geometry and anisotropic initial velocities. This model may be considered as a simplified picture of inhomogeneous particle-emitting sources.

Apart from using viscous hydrodynamics to describing the droplet evolution, we adopt all the ingredients of the granular source model used in Ref. [54]. The initial energy density distribution of a single droplet is assumed to be a Woods-Saxon distribution [54], and the QGP droplets are initially distributed within a cylinder along the beam direction (z-axis) by [53, 54]

$$
\begin{aligned}
\frac{d N_{d}}{d x_{0} d y_{0} d z_{0}} \propto & {\left[1-e^{-\left(x_{0}^{2}+y_{0}^{2}\right) / \Delta \mathcal{R}_{T}^{2}}\right] \theta\left(\mathcal{R}_{T}-\rho_{0}\right) } \\
& \times \theta\left(\mathcal{R}_{z}-\left|z_{0}\right|\right),
\end{aligned}
$$

where $\rho_{0}=\sqrt{x_{0}^{2}+y_{0}^{2}}$ and $z_{0}$ are the initial transverse and longitudinal coordinates of the droplet centers, $\mathcal{R}_{T}$ and $\mathcal{R}_{z}$ describe the initial transverse and longitudinal sizes of the source, and $\Delta \mathcal{R}_{T}$ is a transverse shell parameter. Quantity $\Delta \mathcal{R}_{T}$ is used to describe the effect where blastwave-type expansion in early high density QGP matter may lead to a void in the system central region [6, 62] at the time of the granular source formation $\left(\Delta \mathcal{R}_{T} \rightarrow 0\right.$ for the uniform transverse distribution of droplets). The initial radius of the droplets, $r_{0}$, satisfies a Gaussian distribution with standard deviation $\sigma_{d}$ in the droplet local frame. We take the initial velocities of the droplets in the granular source as 53, 54]

$$
v_{\mathrm{d} i}=\operatorname{sign}\left(r_{0 i}\right) \cdot a_{i}\left(\frac{\left|r_{0 i}\right|}{\mathcal{R}_{i}}\right)^{b_{i}}, \quad i=1,2,3,
$$

where $r_{0 i}$ is $x_{0}, y_{0}$, or $z_{0}$ for $i=1,2$, or 3 , and $\operatorname{sign}\left(r_{0 i}\right)$ denotes the signal of $r_{0 i}$, which ensures an outward droplet velocity. In Eq. (20), $\mathcal{R}_{i}=\left(\mathcal{R}_{T}, \mathcal{R}_{T}, \mathcal{R}_{z}\right)$, $a_{i}=\left(a_{x}, a_{y}, a_{z}\right)$ and $b_{i}=\left(b_{x}, b_{y}, b_{z}\right)$ are the magnitude and exponent parameters in $x, y$, and $z$ directions respectively. It is also convenient to use the equivalent parameters $\bar{a}_{T}=\left(a_{x}+a_{y}\right) / 2$ and $\Delta a_{T}=a_{x}-a_{y}$ instead of $a_{x}$ and $a_{y}$. The parameters $\bar{a}_{T}$ and $\Delta a_{T}$ describe the initial transverse expansion and asymmetric dynamical behavior of the system, respectively. For simplicity, we take $b_{x}=b_{y}=b_{T}$ in calculations. The parameters $b_{T}$ and $b_{z}$ describe the coordinate dependence of exponential power in transverse and longitudinal directions. The parameters $\left(\bar{a}_{T}, \Delta a_{T}, a_{z}, b_{T}, b_{z}\right)$ reflect the anisotropic coordinate-dependence of the droplet velocity caused by the anisotropic pressure gradient in early QGP matter in the granular source model.

\subsection{Pion momentum spectrum and elliptic flow in viscous granular source model}

In high-energy heavy-ion collisions, the invariant momentum distribution of final particles can be written in the form of a Fourier series [85, 86],

$$
E \frac{d^{3} N}{d^{3} p}=\frac{1}{2 \pi} \frac{d^{2} N}{p_{T} d p_{T} d y}\left[1+\sum_{n} 2 v_{n} \cos (n \phi)\right]
$$

where $\phi$ is the particle azimuthal angle with respect to the reaction plane. In Eq. (21), the first term on the 
right is the transverse momentum spectrum in the rapidity interval $d y$, and the second harmonic coefficient $v_{2}$ in the summation is called the elliptic flow.

In Fig. 3, we plot the pion transverse-momentum spectra of granular sources without viscosity (cyan solid lines), with only shear viscosity (red dashed lines), and with both shear and bulk viscosities (blue dot-dashed lines), for $\mathrm{Au}-\mathrm{Au}$ collisions at $\sqrt{s_{N N}}=200 \mathrm{GeV}$ in the centrality intervals $0-5 \%, 10-20 \%$, and $30-50 \%$, and for $\mathrm{Pb}-\mathrm{Pb}$ collisions at $\sqrt{s_{N N}}=2.76 \mathrm{TeV}$ in the centrality intervals $10-20 \%$ and $40-50 \%$. In these centrality intervals, the experimental data of pion transversemomentum spectrum, elliptic flow, and HBT interferometry are all available. The experimental data plotted in Fig. 3 are from the PHENIX and STAR collaborations at the RHIC [87, 88] and the ALICE collaboration at the LHC [89]. In the calculations of the momentum spectra of the granular sources, we take the rapidity cuts $|y|<0.1$ and $|y|<0.5$ to be the same as in the experimental measurements at the RHIC [88] and at the LHC 89], respectively. The granular source parameters are the same as in Ref. [54] for the Woods-Saxon initial energy distribution of droplets (see Set I of Table 1).

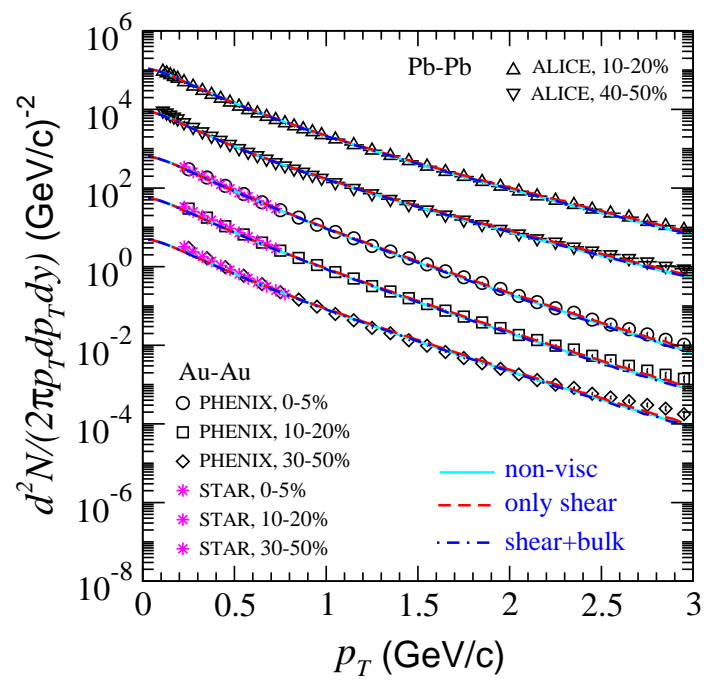

Fig. 3. (Color online) Pion transverse momentum spectra of granular sources without viscosity (cyan solid lines), with only shear viscosity (red dashed lines), and with both shear and bulk viscosities (blue dot-dashed lines), for $\mathrm{Au}-\mathrm{Au}$ collisions at $\sqrt{s_{N N}}=200 \mathrm{GeV}$ and $\mathrm{Pb}-\mathrm{Pb}$ collisions at $\sqrt{s_{N N}}=2.76 \mathrm{TeV}$. The experimental data measured by the PHENIX and STAR collaborations at the RHIC 87, 88] and by the ALICE collaboration at the LHC [89] are also plotted.

One can see from Fig. 3 that the effect of viscosity on the momentum spectrum is very small. In the granular source model, the momentum spectrum depends not only on the droplet evolution velocity, which is viscosity related, but also on the outward motion of the droplet as a whole, which is determined by the velocity parameters $a_{T}$ and $b_{T}$. The outward motion of whole droplet boosts the particles emitted from the droplet. As a result, some particle momenta are decreased and some particle momenta are increased compared to the case where the droplets are at rest in the granular source $\left(a_{T}=0, a_{z}=0\right)$. Although viscosity may lead to a small enhancement of the transverse-momentum distribution of a single droplet at larger $p_{T}$ (see Fig. 2), the outward motion of the whole droplet lets some of the particle contributions in the high transverse momentum region of the single droplet spectrum move to the low transverse momentum region. Meanwhile, it lets some of the particle contributions in the low transverse momentum region of the single droplet spectrum move to the high transverse momentum region. As a result, the outward motion of the whole droplet partially reduces the influence of droplet viscosity on the momentum spectrum in the granular source model.

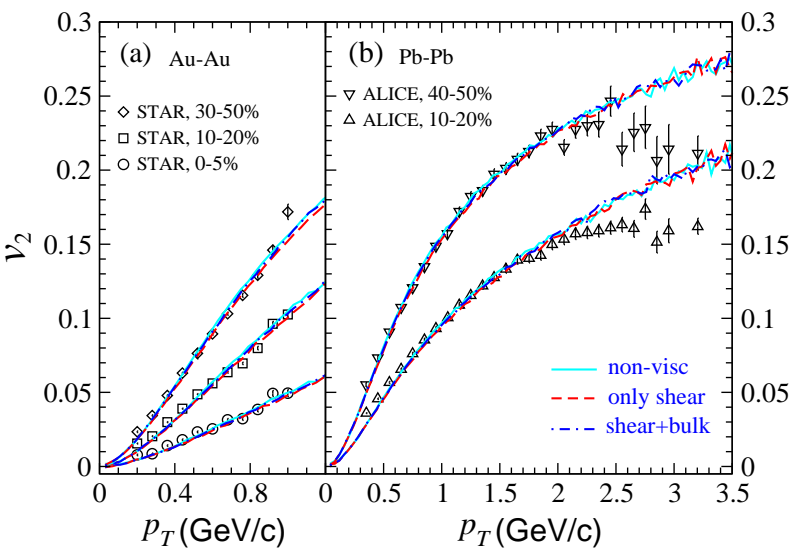

Fig. 4. (Color online) Pion elliptic flow of granular sources without viscosity (cyan solid lines), with only shear viscosity (red dashed lines), and with both shear and bulk viscosities (blue dot-dashed lines), for $\mathrm{Au}-\mathrm{Au}$ collisions at $\sqrt{s_{N N}}=200 \mathrm{GeV}$ [panel (a)] and $\mathrm{Pb}-\mathrm{Pb}$ collisions at $\sqrt{s_{N N}}=2.76$ $\mathrm{TeV}$ [panel (b)], respectively. The experimental data measured by the STAR collaboration at the RHIC 90 and by the ALICE collaboration at the LHC [91, 92] are plotted in panels (a) and (b), respectively.

In Figs. 4(a) and 4(b), we show the results of pion elliptic flow of the granular sources without viscosity (cyan solid lines), with only shear viscosity (red dashed lines), and with both shear and bulk viscosities (blue dot-dashed lines) as in Fig. 3, for Au-Au collisions at $\sqrt{s_{N N}}=200 \mathrm{GeV}$ and $\mathrm{Pb}-\mathrm{Pb}$ collisions at $\sqrt{s_{N N}}=2.76$ $\mathrm{TeV}$, respectively. The experimental data of elliptic flow 
for the $\mathrm{Au}-\mathrm{Au}$ and $\mathrm{Pb}-\mathrm{Pb}$ collisions in the same centrality intervals as the momentum-spectrum data in Fig. 3. measured by the PHENIX and STAR collaborations at the RHIC [87, 88] and the ALICE collaboration at the LHC 89] are also shown in Figs. 廿(a) and 4(b), respectively. We take the pseudorapidity cuts $|\eta|<1.0$ and $|\eta|<0.8$ in the calculations of $v_{2}$ of the granular sources to be the same as in the experimental analyses carried out by the STAR 90 and the ALICE 91, 92 collaborations, respectively. The elliptic flow of the viscous granular source is a little smaller than that of the granular source without viscosity. Because the evolution of the spherical droplet is isotropic, the anisotropic flow $v_{2}$ of the granular source is determined by the velocity parameters $\Delta a_{T}, b_{T}$, and $b_{z}$ [56]. These parameters reflect the anisotropic coordinate-dependence of the droplet velocity caused by the anisotropic pressure gradient in the early QGP matter, and are seldom affected by the droplet evolution afterwards in the granular source model considered. In Ref. [93], the effects of droplet absorption of particles on the momentum spectrum and HBT radii are investigated. Further investigations of the influences of source evolution and absorption on $v_{2}$ in the viscous granular source model will be of interest. At larger $p_{T}(>1.8 \mathrm{GeV} / c)$, the $v_{2}$ results of the granular sources are larger than the experimental data. This is because the current granular source model does not include the hard processes which exist in high-energy heavy-ion collisions.

\subsection{Pion interferometry results of granular sources}

The two-pion HBT correlation function is defined as the ratio of the two-particle momentum spectrum $P\left(\boldsymbol{p}_{1}, \boldsymbol{p}_{2}\right)$ of identical pions to the product of the two single-pion momentum spectra $P\left(\boldsymbol{p}_{1}\right) P\left(\boldsymbol{p}_{2}\right)$. In interferometry analyses in high-energy heavy-ion collisions, the two-pion correlation function is usually fitted by the Gaussian parameterized formula

$$
C\left(q_{\text {out }}, q_{\text {side }}, q_{\text {long }}\right)=1+\lambda e^{-R_{\text {out }}^{2} q_{\text {out }}^{2}-R_{\text {side }}^{2} q_{\text {side }}^{2}-R_{\text {long }}^{2} q_{\text {long }}^{2}},
$$

where $q_{\text {out }}, q_{\text {side }}$, and $q_{\text {long }}$ are the Bertsch-Pratt variables 72, 73], which denote the components of the relative momentum $\boldsymbol{q}=\boldsymbol{p}_{1}-\boldsymbol{p}_{2}$ in the transverse "out" (parallel to the transverse momentum of the pion pair $\boldsymbol{k}_{T}$ ), transverse "side" (in the transverse plane and perpendicular to $\boldsymbol{k}_{T}$ ), and longitudinal ("long") directions, respectively. In Eq. (22), $\lambda$ is the chaoticity parameter, and $R_{\text {out }}, R_{\text {side }}$, and $R_{\text {long }}$ are the HBT radii in the out, side, and long directions respectively.

In experimental HBT analyses, the Coulomb effect on the correlation functions must be considered 94 97]. It becomes the well-known Gamow factor [98] in the case of first-order approximation. Because the Coulomb effect has been carefully removed from the experimental
HBT data 71, 99], we will not consider it in our model HBT analyses. In a more general case, the interference term in the formula of the two-pion correlation function also contains factors concerning two relative momentum products, $e^{-2 R_{\mathrm{os}}^{2} q_{\mathrm{out}} q_{\mathrm{side}}}, e^{-2 R_{\mathrm{ol}}^{2} q_{\mathrm{out}} q_{\mathrm{long}}}$, and $e^{-2 R_{\mathrm{s} 1}^{2} q_{\mathrm{side}} q_{\text {long }}}$ 71, 97, 100 104. They are employed in more detailed HBT analyses. In Ref. 71], the STAR collaboration investigates the dependence of two-pion correlation functions on the particle azimuth angle using a formula containing the factor $e^{-2 R_{\mathrm{os}}^{2} q_{\mathrm{out}} q_{\mathrm{side}}}$, and the relevant Fourier coefficients of HBT correlations are studied as a function of the number of participating nucleons in heavyion collisions at RHIC energy. In Ref. 99], the ALICE collaboration performs two-pion HBT analyses with the correlation-function formula without the factors concerning two relative momentum products in heavy-ion collisions at LHC energy. Because the experimental data of HBT radii $R_{\text {out }}, R_{\text {side }}$, and $R_{\text {long }}$ are available in heavyion collisions at both the RHIC and LHC, we perform the HBT analyses with formula (22) for the granular sources at RHIC and LHC energies and examine the variations of model parameters with collision energy. Further detailed HBT analyses with the more general correlation-function formula for the granular sources will be of interest.

In Fig. 5 we show the results of pion interferometry of the granular sources for $\mathrm{Au}-\mathrm{Au}$ collisions at $\sqrt{s_{N N}}=200$ $\mathrm{GeV}$ and $\mathrm{Pb}-\mathrm{Pb}$ at $\sqrt{s_{N N}}=2.76 \mathrm{TeV}$ in the corresponding centrality intervals as in Fig. 3 The experimental data of the pion interferometry analyses performed by the STAR 71] and the ALICE [99] collaborations are also shown in Fig. 5 by the black solid circles and black solid squares, respectively. In our interferometry analyses for the granular sources, we take the same rapidity or pseudorapidity cuts as in the experimental analyses 71, 99]. In Fig. 5 the results denoted by cyan open circles are for the granular sources without viscosity and with the same model parameters as in Ref. [54] for the Woods-Saxon initial energy distribution of the droplet. The results denoted by red down-triangles and blue uptriangles are for the granular sources with only shear viscosity and with both shear and bulk viscosities, respectively. The parameters for the viscous sources are taken to be the same as for the non-viscous sources 54 . One can see from Fig. 5 that the differences between the results for the granular sources with only shear viscosity and with both shear and bulk viscosities are very small. The main influence of viscosity is on the HBT ra$\operatorname{dius} R_{\text {out }}$, which has an obvious decrease for the viscous sources. The effect of viscosity leads also to the decrease of the ratio $R_{\text {out }} / R_{\text {side }}$. The results of the chaotic parameters $\lambda$ of the granular sources are larger than the experimental data, because many effects in experiments can decrease the measurement value of $\lambda$ 94, 97, 104 106, which exceed our considerations in the granular source model. 


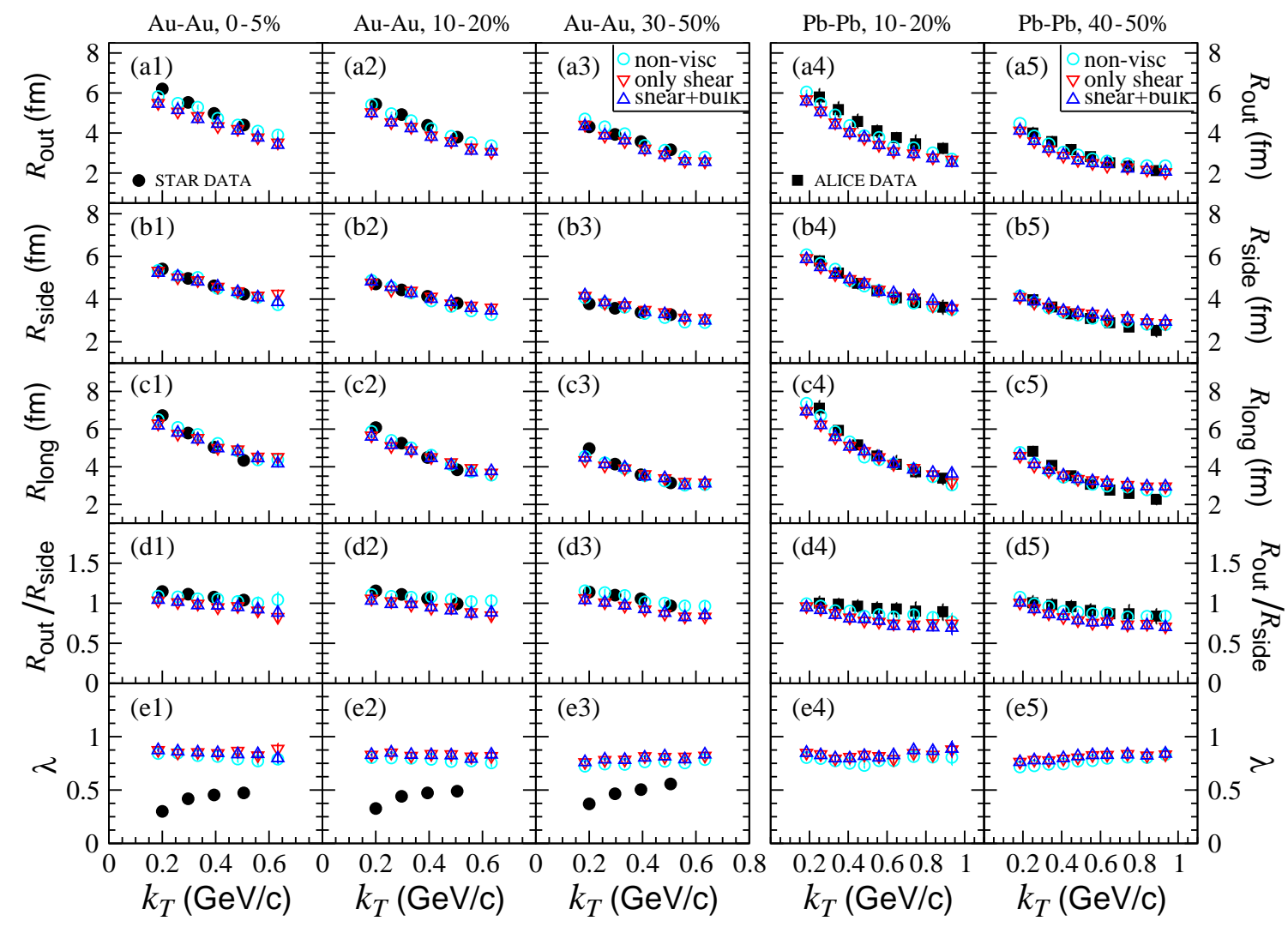

Fig. 5. (Color online) Pion HBT radii and chaotic parameter of the granular sources without viscosity (cyan open circles), with only shear viscosity (red down-triangles), and with both shear and bulk viscosities (blue up-triangles), for $\mathrm{Au}-\mathrm{Au}$ collisions at $\sqrt{s_{N N}}=200 \mathrm{GeV}$ and $\mathrm{Pb}-\mathrm{Pb}$ collisions at $\sqrt{s_{N N}}=2.76 \mathrm{TeV}$ in the same centrality intervals as in Fig. 3 The black solid circles in the left three columns show the experimental data for $\mathrm{Au}-\mathrm{Au}$ collisions measured by the STAR collaboration [71], and the black solid squares in the right two columns show the experimental data for $\mathrm{Pb}-\mathrm{Pb}$ collisions measured by the ALICE collaboration [99].

\section{Influence of viscosity on granular source parameters}

We saw in the last section that the pion transversemomentum spectrum and elliptic flow change little for the granular source with and without viscosity. However, the pion HBT radius $R_{\text {out }}$ of the viscous granular source is obviously smaller than that of the granular source without viscosity. Because $R_{\text {out }}$ is related not only to the source space-geometry but also to the source evolution time and expansion velocity in highenergy heavy-ion collisions [53, 100, 104, 107, 108], it is sensitive to the geometry as well as velocity parameters in the granular source model. The model parameters we used in the last section are fixed for the granular sources without viscosity [54]. In this section, we will determine the model parameters for the viscous granular sources by the experimental data of pion HBT radii as well as pion transverse-momentum spectra and elliptic flow.

Table 1. Adjusted model parameter values and their original values in Ref. 54].

\begin{tabular}{c|cccc|cccc}
\hline & \multicolumn{3}{|c}{ Set II } & \multicolumn{3}{c}{ Set I [54] } \\
& $\sigma_{d}(\mathrm{fm})$ & $\Delta \mathcal{R}_{T}(\mathrm{fm})$ & $\bar{a}_{T}$ & $\Delta a_{T}$ & $\sigma_{d}(\mathrm{fm})$ & $\Delta \mathcal{R}_{T}(\mathrm{fm})$ & $\bar{a}_{T}$ & $\Delta a_{T}$ \\
\hline $\mathrm{Au}-\mathrm{Au}, 0-5 \%$ & 3.0 & 0.21 & 0.445 & 0.069 & 2.5 & 0.70 & 0.469 \\
$\mathrm{Au}-\mathrm{Au}, 10-20 \%$ & 2.8 & 0.15 & 0.434 & 0.126 & 2.5 & 0.50 & 0.457 \\
$\mathrm{Au}-\mathrm{Au}, 30-50 \%$ & 2.5 & 0.00 & 0.408 & 0.167 & 2.5 & 0.30 & 0.453 \\
$\mathrm{~Pb}-\mathrm{Pb}, 10-20 \%$ & 3.0 & 0.27 & 0.471 & 0.095 & 2.5 & 0.90 & 0.122 \\
$\mathrm{~Pb}-\mathrm{Pb}, 40-50 \%$ & 2.5 & 0.00 & 0.412 & 0.130 & 2.5 & 0.40 & 0.496 \\
\hline
\end{tabular}


As viscosity speeds up droplet evolution, on average, we increase the initial droplet radius parameter $\sigma_{d}$ and decrease the initial droplet velocity parameter $\bar{a}_{T}$ for the viscous granular source compared to those for the granular source without viscosity, to counteract the effects of viscosity on the source evolution. The increase of $\sigma_{d}$ leads to an increase of the HBT radius $R_{\text {out }}$ and also an increase of the HBT radius $R_{\text {side }}$. We further decrease the shell parameter $\Delta \mathcal{R}_{T}$ for the viscous granular source to decrease the value of $R_{\text {side }}$, and adjust the velocity parameters $\bar{a}_{T}$ and $\Delta a_{T}$ somewhat to let the results of the momentum spectrum and elliptic flow of the viscous granular sources conform to the experimental data. The other model parameters used for the viscous granular sources are the same as in Ref. 54 for the granular sources without viscosity. In Table 11 we present the adjusted model parameter values (Set II) for the viscous granular sources for heavy-ion collisions of $\mathrm{Au}-\mathrm{Au}$ at $\sqrt{s_{N N}}=200 \mathrm{GeV}$ in the centrality intervals $0-5 \%$, $10-20 \%, 30-50 \%$, and for heavy-ion collisions of $\mathrm{Pb}-\mathrm{Pb}$ at $\sqrt{s_{N N}}=2.76 \mathrm{TeV}$ in the centrality intervals $10-20 \%$ and $40-50 \%$. Meanwhile, the corresponding model parameter values in Ref. 54] (Set I), determined for the granular sources without viscosity, are also presented for comparison.
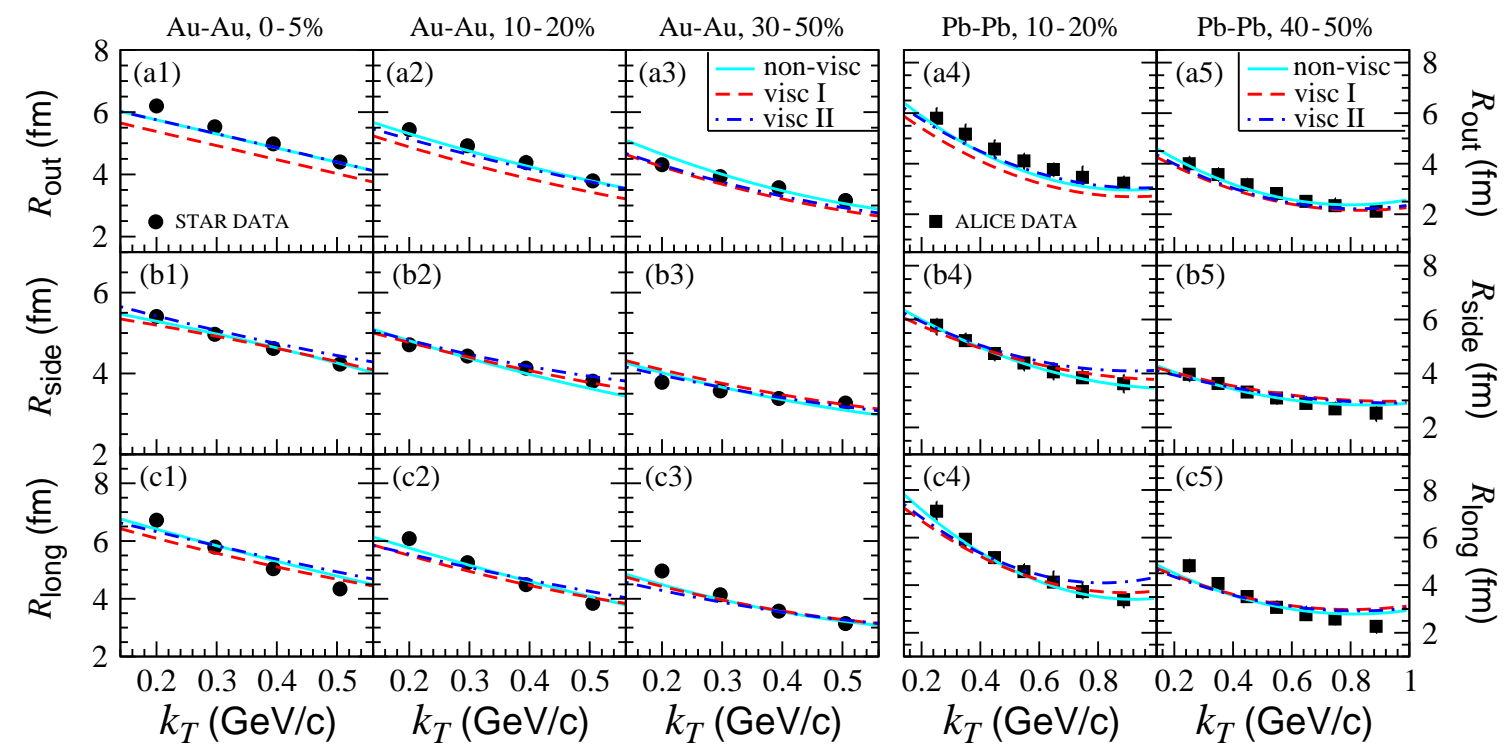

Fig. 6. (Color online) Pion HBT radii of granular sources without viscosity (cyan solid lines) and with viscosity (red dashed and blue dot-dashed lines), for $\mathrm{Au}$-Au collisions at $\sqrt{s_{N N}}=200 \mathrm{GeV}$ and $\mathrm{Pb}-\mathrm{Pb}$ collisions at $\sqrt{s_{N N}}=2.76$ $\mathrm{TeV}$ in the same centrality intervals as in Figs. 3-5. The model parameters used for the solid and dashed lines are the same as in Ref. 54] (parameter set I), and the dot-dashed lines are for the results of viscous granular sources with the adjusted model parameters (parameter set II). The experimental results of the HBT radii of Au-Au collisions measured by the STAR collaboration [71], and $\mathrm{Pb}-\mathrm{Pb}$ collisions measured by the ALICE collaboration [99] are also plotted.

In Fig. 6. we compare the pion HBT radii of viscous granular sources with the adjusted model parameters (visc-II) and with the model parameters as in Ref. [54] for granular sources without viscosity (visc-I). We also compare with the results of the granular source without viscosity (non-visc) and the experimental data of $\mathrm{Au}-\mathrm{Au}$ collisions at $\sqrt{s_{N N}}=200 \mathrm{GeV}$ [71] and $\mathrm{Pb}-\mathrm{Pb}$ collisions at $\sqrt{s_{N N}}=2.76 \mathrm{TeV}$ [99] in the same centrality intervals as in Figs. 3 - 5 . Here, the lines are obtained by fitting the separated results of HBT radii $R\left(k_{T}\right)$ of the granular sources with a binomial of $k_{T}$. The HBT results of $R_{\text {out }}$ for the viscous granular sources with the adjusted parameters are larger than those with the original model parameters [54] and closer to the experimental results. There is not much difference in the results of the HBT radii $R_{\text {side }}$ and $R_{\text {long }}$ for the two sets of model parameters.

We further examine the pion transverse-momentum spectrum and elliptic flow of the granular sources with the model parameter sets I and II. In Fig. 7, we show the results of pion transverse-momentum spectrum [in panel (a)] and elliptic flow [in panels (b) and (c)] of the granular sources with and without viscosity, for $\mathrm{Au}-\mathrm{Au}$ collisions at $\sqrt{s_{N N}}=200 \mathrm{GeV}$ in the centrality intervals $0-5 \%, 10-20 \%$, and $30-50 \%$, and $\mathrm{Pb}-\mathrm{Pb}$ collisions at $\sqrt{s_{N N}}=2.76 \mathrm{TeV}$ in the centrality intervals $10-20 \%$ and $40-50 \%$. Here, the cyan solid lines are for granular 
sources without viscosity and calculated with model parameter set I, the same as in Ref. 54]. The red dashed and blue dot-dashed lines are for viscous granular sources with model parameter sets I and II, respectively. The experimental data of the momentum spectrum and elliptic flow measured by the PHENIX and STAR collaborations at the RHIC [87, 88, 90], and by the ALICE collaboration at the LHC [91, 92] are also plotted. At lower $p_{T}$, the results of the momentum spectra (or elliptic flow) of the granular sources with and without viscosity have little difference, and they are agree with the experimental data. The differences between the granular source results and the experimental data at larger $p_{t}$ are due to the absence of hard processes in the granular source models.
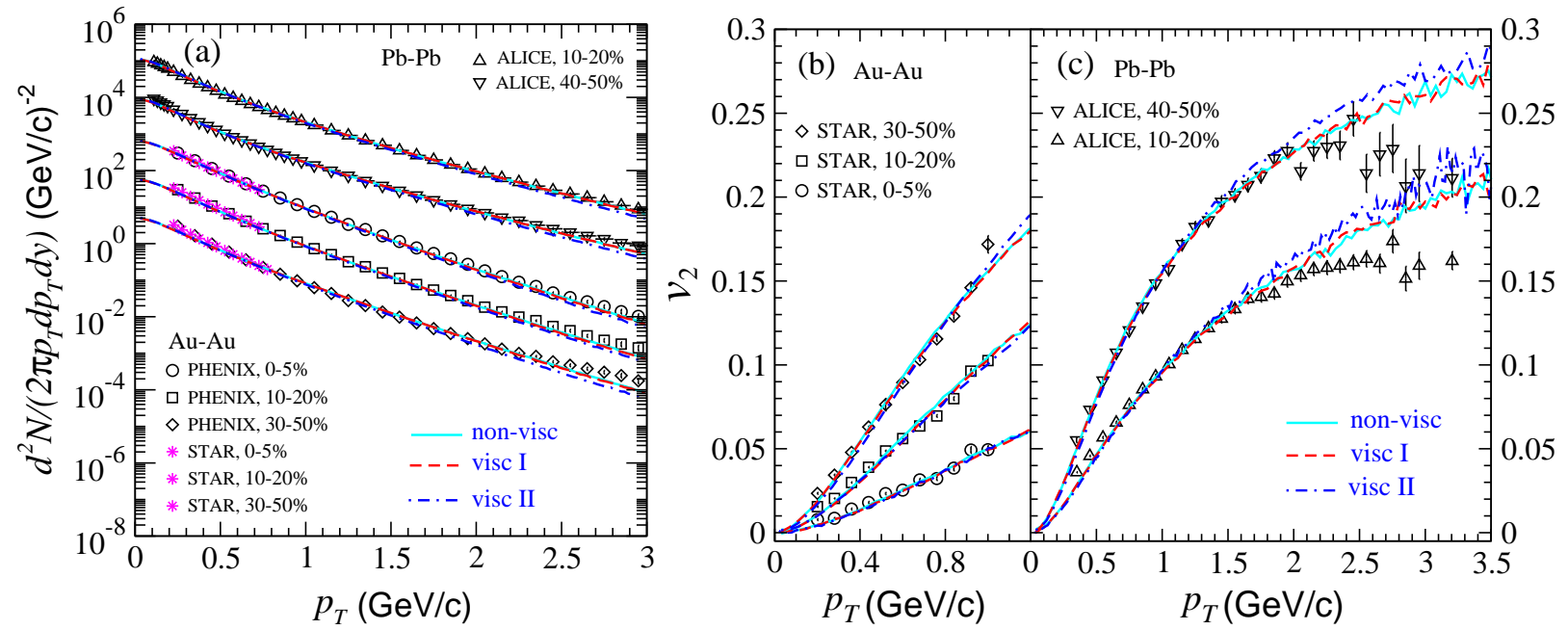

Fig. 7. (Color online) (a) Pion transverse momentum spectra of granular sources without viscosity (cyan solid lines) and with viscosity (red dashed lines and blue dot-dashed lines), for Au-Au collisions at $\sqrt{s_{N N}}=200 \mathrm{GeV}$ and $\mathrm{Pb}-\mathrm{Pb}$ collisions at $\sqrt{s_{N N}}=2.76 \mathrm{TeV}$. The model parameters used for the granular sources without viscosity are the same as in Ref. [54] (parameter set I), and the parameters used for the viscous granular sources are the same as in Ref. 54] (parameter set I) and the adjusted model parameters (parameter set II), respectively. The experimental data measured by the PHENIX and the STAR collaborations at the RHIC 87, 88] and by the ALICE collaboration at the LHC 89] are also plotted. (b) and (c) Pion elliptic flow of the granular sources without viscosity (cyan solid lines) and with viscosity (red dashed lines for model parameter set I and blue dot-dashed lines for model parameter set II)), for $\mathrm{Au}-\mathrm{Au}$ collisions at $\sqrt{s_{N N}}=200 \mathrm{GeV}$ and $\mathrm{Pb}-\mathrm{Pb}$ collisions at $\sqrt{s_{N N}}=2.76 \mathrm{TeV}$, respectively. The experimental data measured by the STAR collaboration at the RHIC [90] and by the ALICE collaboration at the LHC [91, 92] are also plotted in panels (b) and (c), respectively.

In early articles on viscous hydrodynamics, it was pointed out that shear viscosity may lead to the increase in the particle transverse-momentum spectrum at high $p_{T}$ [9, 10, 29, 35], and to the decrease of elliptic flow in high $p_{T}$ regions compared to the ideal hydrodynamic results [12, 28 30, 35]. Also, it was expected that the viscosity might increase the HBT radius $R_{\text {out }}$ and decrease slightly the HBT radius $R_{\text {side }}$ 9, 32]. In the last few years, many groups have utilizes the viscosity effects on the momentum spectrum and elliptic flow to investigate the QGP viscosities, by comparing their model results with experimental data from relativistic heavy-ion collisions 38 40, 45 52]. In Ref. [36], P. Bożek investigated the HBT radii for central heavy-ion collisions in a viscous hydrodynamic model and found that the initial pre-equilibrium flow reduces the HBT radius $R_{\text {out }}$.
It has also been found that the bulk viscosity has very small influence on HBT radii [37.

In the viscous granular source model considered, the outward motion of the droplet reduces the effect of viscosity on transverse-momentum spectra, as mentioned in Sec. 3. Because the elliptic flow in the granular source model is determined by the anisotropic initial velocity of the droplets, the influences of viscosity on the $v_{2}$ values are negligible. As a result, the differences in the model velocity parameters between granular sources with and without viscosity are very small. However, viscosity may increase the HBT radius $R_{\text {out }}$ in the granular source model and therefore lead to changes in the geometry parameters of the viscous granular sources from those of the granular sources without viscosity. One can see from Table1 that the values of $\sigma_{d}$ for the viscous granular sources 
are larger than those for the granular sources without viscosity, except for the most peripheral collisions. Also, the values of the shell parameter of source $\Delta \mathcal{R}_{T}$ of the viscous granular sources are smaller than those of the granular sources without viscosity. These indicate that the viscous granular sources have larger droplets and a less obvious shell-like structure than the granular sources without viscosity. The average number of droplets in a granular source is related to the initial source volume $\mathcal{R}_{T}^{2} \mathcal{R}_{Z}$, the shell parameter $\Delta \mathcal{R}_{T}$, and $\sigma_{d}$. For a uniform distribution of the droplets in a granular source, the average number of droplets in the source is proportional to $\left(\mathcal{R}_{T}^{2} \mathcal{R}_{z} /\left\langle a^{3}\right\rangle\right) \propto\left(\mathcal{R}_{T}^{2} \mathcal{R}_{z} / \sigma_{d}^{3}\right)$, where $\left\langle a^{3}\right\rangle$ denotes the average of the droplet-radius cube over granular sources with the same collision energy and centrality.

\section{Summary and Discussion}

Based on the second-order Müller-Israel-Stewart theory of dissipative hydrodynamics [61], we have given a viscous hydrodynamic description for a spherical QGP droplet and examined its evolution. A granular source model consisting of the viscous QGP droplets has been developed. We have analyzed pion transversemomentum spectra, elliptic flow, and Hanbury-BrownTwiss (HBT) interferometry in the viscous granular source model, for collisions of Au-Au at $\sqrt{s_{N N}}=200 \mathrm{GeV}$ and $\mathrm{Pb}-\mathrm{Pb}$ at $\sqrt{s_{N N}}=2.76 \mathrm{TeV}$ and in different centrality intervals. By comparing the multi-observable results of the granular sources with the experimental data, we have investigated the effects of viscosity on the observables. We find that the shear viscosity of the QGP droplet speeds up the droplet evolution and the influence of the bulk viscosity on the evolution is negligible. Although there are viscous effects on the droplet evolution, the pion transverse-momentum spectrum and elliptic flow change little for granular sources with and without viscosity. This is because the outward motion of droplets in the granular sources reduce the effect of viscosity on the pion momentum spectrum, and the value of elliptic flow is mainly determined by the anisotropic initial velocities of droplets in the granular source model. The crucial effect of droplet viscosity is on the HBT radius $R_{\text {out }}$. It has a significant decrease for viscous granular sources compared to granular sources without viscosity.

In high-energy heavy-ion collisions, transversemomentum spectra, elliptic flow, and HBT correlations are important observables. The experimental data of these observables give strict constraints on the model parameters. Examining the changes in model parameters with collision energy, centrality, and viscosity may provide an integrated picture of source geometry and dynamics. We determine the model parameters of the viscous granular sources by the experimental data of these observables for pions and investigate the effects of viscosity on the model parameters. The results indicate that the viscosity of the droplet leads to an increase in the initial droplet-radius parameter and decreases in the source-shell parameter and the initial droplet velocity in the granular source model. These change the initial picture of a granular source relative to the case without viscosity.

As a simplification of the particle-emitting sources with granular inhomogeneous structures formed in ultrarelativistic heavy-ion collisions, granular source models are used to describe the system evolution after a later stage of QGP expansion. In the models, the QGP lumps are assumed to have spherical geometry for simplicity, and anisotropic pressure in the early QGP matter is assumed to lead to anisotropic initial velocities of the droplets. We determine the model geometry and velocity parameters $\left(\mathcal{R}_{T}, \mathcal{R}_{z}, \sigma_{d}\right)$ and $\left(\bar{a}_{T}, \Delta a_{T}, a_{z}, b_{T}, b_{z}\right)$ by the experimental data of the multi-observables in order to let the model reproduce these experimental observables. The variations of the model parameters with collision centrality and energy exhibit certain regularities. The results of the pion transverse-momentum spectra, elliptic flow, and HBT radii of the granular sources are in accordance with the experimental data of $\mathrm{Au}-\mathrm{Au}$ collisions at $\sqrt{s_{N N}}=200 \mathrm{GeV}$ and $\mathrm{Pb}-\mathrm{Pb}$ collisions at $\sqrt{s_{N N}}=2.76$ $\mathrm{TeV}$ in different centrality intervals. This means that the granular source model, with several evolving centers as the main characteristic, reflects in some degree the physics of the system evolution during the later stages of heavy-ion collisions. The model parameters of the granular sources determined by the experimental data are helpful for the study of the QGP properties and dynamics at earlier stages. Finally, it should be mentioned that the present granular source models involve neither the possible overlap of the droplets evolving later, nor particle rescattering and absorption by other droplets in the source. Further improvements of the granular source model to make it more realistic, and applying it to other hadron observable analyses, such as kaons, will be of interest.

The authors would like to thank M. J. Efaaf, Z. Q. Su, L. Cheng, and H. C. Song for their helpful discussions. 


\section{Appendix A: Derivation for relaxation equation}

Here, we give details of how to derive the relaxation equations for shear viscosity in Eq. (7) from Eq. (4).

\section{First term of Eq. (4)}

The first term of Eq. (4) can be expanded as

$$
\begin{aligned}
\tau_{\pi} \Delta^{\mu \alpha} \Delta^{\nu \beta} D \pi_{\alpha \beta} & =\tau_{\pi}\left(g^{\mu \alpha}-u^{\mu} u^{\alpha}\right)\left(g^{\nu \beta}-u^{\nu} u^{\beta}\right) D \pi_{\alpha \beta} \\
& =\tau_{\pi} g^{\mu \alpha} g^{\nu \beta} D \pi_{\alpha \beta}-\tau_{\pi} g^{\mu \alpha} u^{\nu} u^{\beta} D \pi_{\alpha \beta}-\tau_{\pi} u^{\mu} u^{\alpha} g^{\nu \beta} D \pi_{\alpha \beta}-\tau_{\pi} u^{\mu} u^{\alpha} u^{\nu} u^{\beta} D \pi_{\alpha \beta} .
\end{aligned}
$$

With the relations $D g_{\mu \nu}=D g^{\mu \nu}=0$ and the orthogonality $u_{\mu} \pi^{\mu \nu}=u^{\mu} \pi_{\mu \nu}=0$, we have

$$
\left\{\begin{aligned}
g^{\mu \alpha} g^{\nu \beta} D \pi_{\alpha \beta} & =g^{\nu \beta}\left[D\left(g^{\mu \alpha} \pi_{\alpha \beta}\right)-\left(D g^{\mu \alpha}\right) \pi_{\alpha \beta}\right]=g^{\nu \beta}\left(D \pi_{\beta}^{\mu}\right), \\
g^{\mu \alpha} u^{\nu} u^{\beta} D \pi_{\alpha \beta} & =g^{\mu \alpha} u^{\nu}\left[D\left(u^{\beta} \pi_{\alpha \beta}\right)-\left(D u^{\beta}\right) \pi_{\alpha \beta}\right]=-g^{\mu \alpha} u^{\nu}\left(D u^{\beta}\right) \pi_{\alpha \beta}, \\
u^{\mu} u^{\alpha} g^{\nu \beta} D \pi_{\alpha \beta} & =g^{\nu \beta} u^{\mu}\left[D\left(u^{\alpha} \pi_{\alpha \beta}\right)-\left(D u^{\alpha}\right) \pi_{\alpha \beta}\right]=-g^{\nu \beta} u^{\mu}\left(D u^{\alpha}\right) \pi_{\alpha \beta}, \\
u^{\mu} u^{\alpha} u^{\nu} u^{\beta} D \pi_{\alpha \beta} & =u^{\mu} u^{\nu}\left[u^{\alpha} D\left(u^{\beta} \pi_{\alpha \beta}\right)-\left(D u^{\beta}\right) u^{\alpha} \pi_{\alpha \beta}\right]=0 .
\end{aligned}\right.
$$

Therefore, the first term of Eq. (4) can be expressed as

$$
\tau_{\pi} \Delta^{\mu \alpha} \Delta^{\nu \beta} D \pi_{\alpha \beta}=\tau_{\pi}\left[g^{\nu \beta}\left(D \pi_{\beta}^{\mu}\right)+I^{\mu \nu}\right]
$$

where

$$
I^{\mu \nu} \equiv g^{\mu \alpha} u^{\nu}\left(D u^{\beta}\right) \pi_{\alpha \beta}+g^{\nu \beta} u^{\mu}\left(D u^{\alpha}\right) \pi_{\alpha \beta} .
$$

For a symmetrical sphere, $u^{\theta}=u^{\phi}=0$, so $I^{\theta \theta}=I^{\phi \phi}=0$. The non-vanishing components of $I^{\mu \nu}$ are as follows,

$$
\left\{\begin{aligned}
I^{t t} & =g^{t \alpha} u^{t}\left(D u^{\beta}\right) \pi_{\alpha \beta}+g^{t \beta} u^{t}\left(D u^{\alpha}\right) \pi_{\alpha \beta} \\
& =2 g^{t \alpha} u^{t}\left(D u^{\beta}\right) \pi_{\alpha \beta}=2 g^{t t} u^{t}\left(D u^{t}\right) \pi_{t t}+2 g^{t t} u^{t}\left(D u^{r}\right) \pi_{t r}=-2 \gamma^{4} v_{r}\left(D v_{r}\right) \tau^{r r}, \\
I^{t r} & =g^{t \alpha} u^{r}\left(D u^{\beta}\right) \pi_{\alpha \beta}+g^{r \beta} u^{t}\left(D u^{\alpha}\right) \pi_{\alpha \beta} \\
& =g^{t t} u^{r}\left(D u^{t}\right) \pi_{t t}+g^{t t} u^{r}\left(D u^{r}\right) \pi_{t r}+g^{r r} u^{t}\left(D u^{t}\right) \pi_{t r}+g^{r r} u^{t}\left(D u^{r}\right) \pi_{r r}=-\gamma^{4}\left[v_{r}^{2}+1\right]\left(D v_{r}\right) \tau^{r r}, \\
I^{r t} & =g^{r \alpha} u^{t}\left(D u^{\beta}\right) \pi_{\alpha \beta}+g^{t \beta} u^{r}\left(D u^{\alpha}\right) \pi_{\alpha \beta}=I^{t r} \\
I^{r r} & =g^{r \alpha} u^{r}\left(D u^{\beta}\right) \pi_{\alpha \beta}+g^{r \beta} u^{r}\left(D u^{\alpha}\right) \pi_{\alpha \beta} \\
& =2 g^{r \alpha} u^{r}\left(D u^{\beta}\right) \pi_{\alpha \beta}=2 g^{r r} u^{r}\left(D u^{t}\right) \pi_{r t}+2 g^{r r} u^{r}\left(D u^{r}\right) \pi_{r r}=-2 \gamma^{4} v_{r}\left(D v_{r}\right) \tau^{r r}=I^{t t} .
\end{aligned}\right.
$$

Here we use the relations

$$
\gamma=\frac{1}{\sqrt{1-v_{r}^{2}}}, \quad v_{r} \frac{\partial\left(\gamma v_{r}\right)}{\partial t}=\frac{\partial \gamma}{\partial t}, \quad v_{r} \frac{\partial\left(\gamma v_{r}\right)}{\partial r}=\frac{\partial \gamma}{\partial r}
$$

Therefore, we have

$$
\begin{aligned}
& \tau_{\pi} \Delta^{t \alpha} \Delta^{t \beta} D \pi_{\alpha \beta}=\tau_{\pi}\left[g^{t \beta}\left(D \pi_{\beta}^{t}\right)+I^{t t}\right]=\tau_{\pi}\left[g^{t t}\left(D \pi_{t}^{t}\right)+I^{t t}\right]=\tau_{\pi} \gamma^{2} v_{r}^{2}\left(D \tau^{r r}\right), \\
& \tau_{\pi} \Delta^{t \alpha} \Delta^{r \beta} D \pi_{\alpha \beta}=\tau_{\pi}\left[g^{t \beta}\left(D \pi_{\beta}^{r}\right)+I^{t r}\right]=\tau_{\pi}\left[g^{t t}\left(D \pi^{r}{ }_{t}\right)+I^{t r}\right]=\tau_{\pi} \gamma^{2} v_{r}\left(D \tau^{r r}\right), \\
& \tau_{\pi} \Delta^{r \alpha} \Delta^{t \beta} D \pi_{\alpha \beta}=\tau_{\pi}\left[g^{r \beta}\left(D \pi_{\beta}^{t}\right)+I^{r t}\right]=\tau_{\pi}\left[g^{r r}\left(D \pi_{r}^{t}\right)+I^{r t}\right]=\tau_{\pi} \Delta^{t \alpha} \Delta^{r \beta} D \pi_{\alpha \beta}, \\
& \tau_{\pi} \Delta^{r \alpha} \Delta^{r \beta} D \pi_{\alpha \beta}=\tau_{\pi}\left[g^{r \beta}\left(D \pi_{\beta}^{r}\right)+I^{r r}\right]=\tau_{\pi}\left[g^{r r}\left(D \pi_{r}^{r}\right)+I^{r r}\right]=\tau_{\pi} \gamma^{2}\left(D \tau^{r r}\right), \\
& \tau_{\pi} \Delta^{\theta \alpha} \Delta^{\theta \beta} D \pi_{\alpha \beta}=\tau_{\pi}\left[g^{\theta \beta}\left(D \pi_{\beta}^{\theta}\right)\right]=\tau_{\pi}\left[g^{\theta \theta}\left(D \pi_{\theta}^{\theta}\right)\right]=\tau_{\pi} \frac{1}{r^{2}}\left(D \tau^{\theta \theta}\right), \\
& \tau_{\pi} \Delta^{\phi \alpha} \Delta^{\phi \beta} D \pi_{\alpha \beta}=\tau_{\pi}\left[g^{\phi \beta}\left(D \pi_{\beta}^{\phi}\right)\right]=\tau_{\pi}\left[g^{\phi \phi}\left(D \pi_{\phi}^{\phi}\right)\right]=\tau_{\pi} \frac{1}{r^{2} \sin ^{2} \theta}\left(D \tau^{\phi \phi}\right),
\end{aligned}
$$

where $D=\gamma\left(\partial_{t}+v_{r} \partial_{r}\right)$.

\section{Second term of Eq. (4)}

For a spherical system, we have

$$
\pi^{\mu \nu}=\left(\begin{array}{cccc}
\gamma^{2} v_{r}^{2} \tau^{t t} & \gamma^{2} v_{r} \tau^{t r} & 0 & 0 \\
\gamma^{2} v_{r} \tau^{r t} & \gamma^{2} \tau^{r r} & 0 & 0 \\
0 & 0 & \frac{1}{r^{2}} \tau^{\theta \theta} & 0 \\
0 & 0 & 0 & \frac{1}{r^{2} \sin ^{2} \theta} \tau^{\phi \phi}
\end{array}\right)
$$


where $\tau^{t t}=\tau^{t r}=\tau^{r t}=\tau^{r r}$ and $\tau^{\theta \theta}=\tau^{\phi \phi}$.

$$
\begin{aligned}
& \pi_{\alpha \beta}=g_{\alpha \mu} \pi^{\mu \nu} g_{\nu \beta} \\
& =\left(\begin{array}{cccc}
1 & 0 & 0 & 0 \\
& -1 & 0 & 0 \\
& 0 & -r^{2} & 0 \\
& 0 & 0 & -r^{2} \sin ^{2} \theta
\end{array}\right)\left(\begin{array}{cccc}
\gamma^{2} v_{r}^{2} \tau^{r r} & \gamma^{2} v_{r} \tau^{r r} & 0 & 0 \\
\gamma^{2} v_{r} \tau^{r r} & \gamma^{2} v_{r} \tau^{r r} & 0 & 0 \\
0 & 0 & \frac{1}{r^{2}} \tau^{\theta \theta} & 0 \\
0 & 0 & 0 & 0 \frac{1}{r^{2} \sin ^{2} \theta} \tau^{\phi \phi}
\end{array}\right)\left(\begin{array}{cccc}
1 & 0 & 0 & 0 \\
-1 & 0 & 0 \\
0 & -r^{2} & 0 \\
0 & 0 & -r^{2} \sin ^{2} \theta
\end{array}\right) \\
& =\left(\begin{array}{cccc}
\gamma^{2} v_{r}^{2} \tau^{r r} & -\gamma^{2} v_{r} \tau^{r r} & 0 & 0 \\
-\gamma^{2} v_{r} \tau^{r r} & \gamma^{2} \tau^{r r} & 0 & 0 \\
0 & 0 & r^{2} \tau^{\theta \theta} & 0 \\
0 & 0 & 0 & r^{2} \sin ^{2} \theta \tau^{\phi \phi}
\end{array}\right) \\
& \pi_{\nu}^{\mu}=g^{\mu \lambda} \pi_{\lambda \nu} \\
& =\left(\begin{array}{cccc}
1 & 0 & 0 & 0 \\
& -1 & 0 & 0 \\
& 0 & -\frac{1}{r^{2}} & 0 \\
& 0 & -\frac{1}{r^{2} \sin ^{2} \theta}
\end{array}\right)\left(\begin{array}{cccc}
\gamma^{2} v_{r}^{2} \tau^{r r} & -\gamma^{2} v_{r} \tau^{r r} & 0 & 0 \\
-\gamma^{2} v_{r} \tau^{r r} & \gamma^{2} \tau^{r r} & 0 & 0 \\
0 & 0 & r^{2} \tau^{\theta \theta} & 0 \\
0 & 0 & 0 & r^{2} \sin ^{2} \theta \tau^{\phi \phi}
\end{array}\right) \\
& =\left(\begin{array}{cccc}
\gamma^{2} v_{r}^{2} \tau^{r r} & -\gamma^{2} v_{r} \tau^{r r} & 0 & 0 \\
\gamma^{2} v_{r} \tau^{r r} & -\gamma^{2} \tau^{r r} & 0 & 0 \\
0 & 0 & -\tau^{\theta \theta} & 0 \\
0 & 0 & 0 & -\tau^{\phi \phi}
\end{array}\right) .
\end{aligned}
$$

As $\pi^{\mu \nu}$ is traceless, i.e. $\pi_{\mu}^{\mu}=0$, so $\tau^{r r}+\tau^{\theta \theta}+\tau^{\phi \phi}=0$.

\section{Third term of Eq. (4)}

For the third term of Eq. (4), we have

$$
\begin{aligned}
\nabla^{<\mu} u^{\nu>} & \equiv \nabla^{(\mu} u^{\nu)}-\frac{1}{3} \Delta^{\mu \nu} d_{\lambda} u^{\lambda} \\
& =\frac{1}{2}\left(\nabla^{\mu} u^{\nu}+\nabla^{\nu} u^{\mu}\right)-\frac{1}{3} \Delta^{\mu \nu} \Theta,
\end{aligned}
$$

and

$$
\nabla^{\mu} u^{\alpha}=\Delta^{\mu \nu} d_{\nu} u^{\alpha}=\Delta^{\mu \nu}\left(\partial_{\nu} u^{\alpha}+\Gamma_{\nu \lambda}^{\alpha} u^{\lambda}\right),
$$

where $\Theta=d_{\lambda} u^{\lambda}$, and we further define $\tilde{\theta} \equiv \partial_{\lambda} u^{\lambda}$. Then, we have

$$
\begin{aligned}
& \nabla^{\mu} u^{\alpha}=\left(\begin{array}{cccc}
\nabla^{t} u^{t} & \nabla^{t} u^{r} & 0 & \\
\nabla^{r} u^{t} & \nabla^{r} u^{r} & 0 & \\
0 & 0 & \nabla^{\theta} u^{\theta} & 0 \\
0 & 0 & 0 & \nabla^{\phi} u^{\phi}
\end{array}\right)=\left(\begin{array}{cccc}
-\gamma^{2} v_{r}^{2} \tilde{\theta} & -\gamma^{2} v_{r} \tilde{\theta} & 0 & 0 \\
-\gamma^{2} v_{r} \tilde{\theta} & -\gamma^{2} \tilde{\theta} & 0 & 0 \\
0 & 0 & -\frac{1}{r^{3}} \gamma v_{r} & 0 \\
0 & 0 & 0 & -\frac{1}{r^{3} \sin ^{2} \theta} \gamma v_{r}
\end{array}\right) \text {, } \\
& \nabla^{\langle\mu} u^{\nu\rangle}=\left(\begin{array}{cccc}
\nabla^{\langle t} u^{t\rangle} & \nabla^{\langle t} u^{r\rangle} & 0 & 0 \\
\nabla^{\langle r} u^{t\rangle} & \nabla^{\langle r} u^{r\rangle} & 0 & 0 \\
0 & 0 & \nabla^{\langle\theta} u^{\theta\rangle} & 0 \\
0 & 0 & 0 & \nabla^{\langle\phi} u^{\phi\rangle}
\end{array}\right)=\left(\begin{array}{cccc}
\gamma^{2} v_{r}^{2} \sigma^{r r} & \gamma^{2} v_{r} \sigma^{r r} & 0 & 0 \\
\gamma^{2} v_{r} \sigma^{r r} & \gamma^{2} \sigma^{r r} & 0 & 0 \\
0 & 0 & \frac{1}{r^{2}} \sigma^{\theta \theta} & 0 \\
0 & 0 & 0 & \frac{1}{r^{2} \sin ^{2} \theta} \sigma^{\phi \phi}
\end{array}\right),
\end{aligned}
$$

where

$$
\left\{\begin{array}{l}
\sigma^{t t}=\sigma^{t r}=\sigma^{r t}=\sigma^{r r}=\left[-\theta+\frac{1}{3} \Theta\right] \\
\sigma^{\theta \theta}=\sigma^{\phi \phi}=\left[-\gamma \frac{v_{r}}{r}+\frac{1}{3} \Theta\right]
\end{array} \Rightarrow \sigma^{r r}=-2 \sigma^{\theta \theta}=-2 \sigma^{\phi \phi} .\right.
$$

\section{Last term of Eq. (4)}


The last term of Eq. (4) can be written as

$$
\frac{1}{2} \pi^{\mu \nu} \tilde{\eta} T d_{\lambda}\left(\frac{\tau_{\pi} u^{\lambda}}{\tilde{\eta} T}\right)=\frac{1}{2} \pi^{\mu \nu} \tilde{\eta} T\left[\frac{\tau_{\pi}}{\tilde{\eta} T} d_{\lambda} u^{\lambda}+u^{\lambda} d_{\lambda}\left(\frac{\tau_{\pi}}{\tilde{\eta} T}\right)\right]=\frac{1}{2} \pi^{\mu \nu} \tilde{\eta} T\left[\frac{\tau_{\pi}}{\tilde{\eta} T} \Theta+D\left(\frac{\tau_{\pi}}{\tilde{\eta} T}\right)\right] .
$$

Now, one can obtain the shear-viscosity related differential equations in Eq. (7) from the above derivations.

\section{References}

1 D. J. Gross, R. D. Pisarski, and L. G. Yaffe, Rev. Mod. Phys., 53: $43(1981)$

2 I. Arsene et al (BRAHMS collaboration), Nucl. Phys. A, 757: $1(2005)$

3 B. B. Back et al (PHOBOS collaboration), Nucl. Phys. A, 757: $28(2005)$

4 J. Adams et al (STAR collaboration), Nucl. Phys. A, 757: 102 (2005)

5 K. Adcox et al (PHENIX collaboration), Nucl. Phys. A, 757: $184(2005)$

6 D. H. Rischke, arXiv:nucl-th/9809044

7 P. F. Kolb and U. Heinz, arXiv:nucl-th/0305084

8 Y. Hama, T. Kodama, O. Socolowski Jr, Braz. J. Phys., 35: 24 (2005); arXiv:hep-ph/0407264

9 A. Muronga and D. H. Rischke, arXiv:nucl-th/0407114

10 R. Baier, P. Romatschke, and U. A. Wiedemann, Phys. Rev. C, 73: 064903 (2006)

11 U. Heinz, H. C. Song, and A. K. Chaudhuri, Phys. Rev. C, 73 $034904(2006)$

12 H. C. Song and U. Heinz, Phys. Rev. C, 77: 064901 (2008)

13 B. Schenke, S. Jeon, and C.Gale, Phys. Rev. C, 82: 014903 (2010)

14 A. Monnai and T. Hirano, Nucl. Phys. A, 847: 283 (2010)

15 P. Romatschke, Int. J. Mod. Phys. E, 19: 1 (2010)

16 H. C. Song, S. A. Bass, U. Heinz, T. Hirano, and C. Shen, Phys. Rev. Lett., 106: 192301 (2011)

17 P. Huovinen, Int. J. Mod. Phys. E, 22: 1330029 (2013)

18 C. Gale, S. Jeon, and B. Schenke, Int. J. of Mod. Phys. A, 28: 1340011 (2013)

19 S. Jeon and U. Heinz, arXiv:1503.03931

20 R. Derradi de Souza, T. Koide, T. Kodama, Prog. Part. Nucl. Phys., 86: 35 (2016); arXiv:1506.03863

21 M. Gyulassy, Structure and Dynamics of Elementary Matter (Dordrecht: Kluwer, 2004), p. 159

22 P. Huovinen, Quark Gluon Plasma 3 (Singapore: World Scientific, 2004), p. 600; P. F. Kolb and U. Heinz, ibid, p. 634

23 M. Gyulassy and L. McLerran, Nucl. Phys. A 750: 30 (2005)

24 E. V. Shuryak, Nucl. Phys. A 750: 64 (2005)

25 B. Muller and J. L. Nagle, Annu. Rev. Nucl. Part. Sci. 56: 93 (2006)

26 A. K. Chaudhuri, Phys. Rev. C 74: 044904 (2006)

27 A. Muronga, Phys. Rev. C 76: 014909; ibid 014910 (2007)

28 P. Romatschke and U. Romatschke, Phys. Rev. Lett. 99: 172301 (2007)

29 K. Dusling and D. Teaney, Phys. Rev. C 77: 034905 (2008)

30 M. Luzum and P. Romatschke, Phys. Rev. C 78: 034915 (2008) [Erratum, ibid 79: 039903] (2009)

31 M. Luzum and P. Romatschke, Phys. Rev. Lett. 103: 262302 (2009)

32 S. Pratt and J. Vredevoogd, Phys. Rev. C 78: 054906 (2008)

33 S. Pratt, Phys. Rev. Lett. 102: 232301 (2009)

34 A. Monnai and T. Hirano, Phys. Rev. C 80: 054906 (2009)

35 H. C. Song and U. Heinz, Phys. Rev. C 81: 024905 (2010)

36 P. Bożek, Phys. Rev. C 83: 044910 (2011)

37 P. Bożek and I. Wyskiel-Piekarska, Phys. Rev. C 85: 064915 (2012)
38 C. Shen, U. Heinz, P. Huovinen, and H. C. Song, Phys. Rev. C 82: 054904 (2010)

39 B. Schenke, S. Jeon, and C. Gale, Phys. Rev. Lett. 106: 042301 (2011)

40 H. Song, S. A. Bass, U. Heinz, T. Hirano, C. Shen, Phys. Rev. C 83: 054910 (2011)

41 V. Roy and A. K. Chaudhuri, Phys. Rev. C 85: 024909 (2012)

42 K. Dusling and T. Schäfer, Phys. Rev. C 85: 044909 (2012)

43 A. K. Chaudhuri, Advances in High Energy Physics 2013: $693180(2013)$

44 G. Vujanovic, C. Young, B. Schenke et al, Phys. Rev. C 89: $034904(2014)$

45 U. Heinz and R. Snellings, Annu. Rev. Nucl. Part. Sci 63: 123 (2013)

46 M. Ruggieri, F. Scardina, S. Plumari, and V. Greco, Phys. Rev. C 89: 054914 (2014)

47 H. Song, S. A. Bass, and U. Heinz, Phys. Rev. C 83: 054912 (2011)

48 C. Shen, U. Heinz, P. Huovinen, and H. Song, Phys. Rev. C 84: 044903 (2011)

49 B. Schenke, S. Jeon, C. Galeb, Phys. Lett. B 702: 59 (2011)

50 C. Shen and U. Heinz, Phys. Rev. C 85: 054902 (2012)

51 R. A. Soltz, I. Garishvili, M. Cheng et al, Phys. Rev. C 87: 044901 (2013)

52 Iu. A. Karpenko, P. Huovinen, H. Petersen, and M. Bleicher, Phys. Rev. C 91: 064901 (2015)

53 J. Yang, Y. Y. Ren, and W. N. Zhang, Advances in High Energy Physics 2015: 846154 (2015)

54 J. Yang, Y. Y. Ren, and W. N. Zhang, in Proceeding of the Xth Workshop on Particle Correlations and Femtoscopy (WPCF14) at Gyöngyös, Hungary on Aug. 2014; arXiv:1501.03651

55 W. N. Zhang, M. J. Efaaf, and C. Y. Wong, Phys. Rev. C 70: $024903(2004)$

56 W. N. Zhang, Y. Y. Ren, and C. Y. Wong, Phys. Rev. C 74: 024908 (2006)

57 W. N. Zhang, Z. T. Yang, and Y. Y. Ren, Phys. Rev. C 80: 044908 (2009)

58 W. N. Zhang, H. J. Yin, and Y. Y. Ren, Chin. Phys. Lett. 28: 122501 (2011)

59 W. N. Zhang, in Proceedings of the 7th Workshop on Particle Correlations and Femtoscope (WPCF'11), Pos no. 051, Tokyo, Japan, Sep. 2011; arXiv:1202.6560

60 L. D. Landau and E. M. Lifshitz, in Fluid Mechanics (Pergamon: Oxford, 1963)

61 I. Müller, Z. Phys. 198: 329 (1967); W. Israel, Ann. Phys. 100: 310 (1976); J. M. Stewart, Proc. Roy. Soc. A 357: 59 (1977); W. Israel and J. M. Stewart, Ann. Phys 118: 341 (1979)

62 D. H. Rischke, S. Bernard, and J. A. Maruhn, Nucl. Phys. A 595: 346 (1995); D. H. Rischke and M. Gyulassy, Nucl. Phys. A 608: 479 (1996)

63 W. N. Zhang, M. J. Efaaf, C. Y. Wong, and M. Khaliliasr, Chin. Phys. Lett. 10: 1918 (2004); M. J. Efaaf, W. N. Zhang, M. Khaliliasr, E. P. Jin, and Y. M. Liu, High Energy Phys. Nucl. Phys. 29: 46 (2005); M. J. Efaaf, W. N. Zhang, M. Khaliliasr, L. Huo, E. P. Jin, and J. B. Zhang, High Energy Phys. Nucl. Phys. 29: 467 (2005)

64 Y. Hu, W. N. Zhang, and Y. Y. Ren, J. Phys. G 42: 045106 (2015) 
65 P. Kovtun, D. T. Son, and A. O. Starinets, Phys. Rev. Lett. 94: 111601 (2005).

66 A. Buchel, Phys. Lett. B 663: 286 (2008)

67 F. Cooper and G. Frye, Phys. Rev. D 10: 186 (1974)

68 C. Adler et al (STAR Collaboration), Phys. Rev. Lett. 87: 082301 (2001)

69 K. Adcox et al (PHENIX Collaboration), Phys. Rev. Lett. 88: $192302(2002)$

70 S. S. Adler et al. (PHENIX Collaboration), Phys. Rev. Lett. 93: 152302 (2004)

71 J. Adams et al (STAR Collaboration), Phys. Rev. C 71: 044906 (2005)

72 G. Bertsch, M. Gong, and M. Tohyama, Phys. Rev. C 37: 1896 (1988); G. Bertsch, Nucl. Phys. A 498: 173c (1989)

73 S. Pratt, T. Csörgő, and J. Zimányi, Phys. Rev.C 42: 2646 (1990)

74 E. Witten, Phys. Rev. D 30: 272 (1984)

75 L. P. Csernai, J. I. Kapusta, Phys. Rev. D 46: 1379 (1992); Phys. Rev. Lett. 69: 737 (1992)

76 R. Venugopalan and A. P. Vischer, Phys. Rev. E 49: 5849 (1994)

77 J. Randrup, Phys. Rev. Lett. 92: 122301 (2004)

78 Y. Y. Ren, W. N. Zhang, J. L. Liu, Phys. Lett. B 669: 317 (2008)

79 Z. T. Yang, W. N. Zhang, L. Huo, J. B. Zhang, J. Phys. G 39 : $015113(2009)$

80 G. Torrieri, B. Tomášik, I. Mishustin, Phys. Rev. C 77: 034903 (2008)

81 J. Takahashi, B. M. Tavares, W. L. Qian et al, Phys. Rev. Lett. 103: 242301 (2009)

82 K. Werner, Iu. Karpenko, T. Pierog, M. Bleicher, and K. Mikhailov, Phys. Rev. C 82: 044904 (2010)

83 B. Schenke, S. Jeon, C. Gale, Phys. Rev. Lett. 106: 042301 (2011)

84 A. Adare, M. Luzum, H. Petersen, Phys. Scripta 87: 048001 (2013); arXiv:1212.5388 [nucl-th].

85 S. Voloshin and Y. Zhang, Z. Phys. C 70: 665 (1996)

86 A. M. Poskanzer and S. A. Voloshin, Phys. Rev. C 58: 1671 (1998)

87 S. S. Adler et al (PHENIX Collaboration), Phys. Rev. C 69:
$034909(2004)$

88 J. Adams et al (STAR Collaboration), Phys. Rev. Lett. 92: 112301 (2004)

89 B. Abelev et al (ALICE Collaboration), Phys. Rev. C 88: 044910 (2013).

90 J. Adams et al. (STAR Collaboration), Phys. Rev. C 72, 014904 (2005).

91 R. Snellings (for ALICE Collaboration), J. Phys. G 38: 124013 (2011); M. Krzewicki (for ALICE Collaboration), J. Phys. G 38: 124047 (2011)

92 F. Noferini et al (ALICE Collaboration), Nucl. Phys. A 904: 483c (2013)

93 J. Yang and W. N. Zhang, Nucl. Sci. Tech. 27: 147 (2016)

94 M. Gyulassy, S. K. Kauffmann, and Lance W. Wilson, Phys. Rev. C 20: 2267 (1979)

95 S. Pratt, Phys. Rev. D 33: 72 (1986)

96 Y. M. Liu, D. Beavis, S. Y. Chu et al, Phys. Rev. C 34: 1667 (1986)

97 M. A. Lisa, S. Pratt, R. Soltz, and U. Wiedemann, Annu. Rev. Nucl. Part. Sci. 55: 357 (2005)

98 L. I. Schiff, Quantum Mechanics (New York: McGRAW-HILL Book Company, 1955), Sec. 21

99 J. Adam et al (ALICE Collaboration), Phys. Rev. C 93: 024905 (2016)

100 S. Chapman, P. Scotto, and U. Heinz, Phys. Rev. Lett. 74: 4400 (1995)

101 S. Chapman, R. Nix, and U. Heinz, Phys. Rev. C 52: 2694 (1995)

102 M. A. Lisa et al (E895 Collaboration), Phys. Rev. Lett. 84: 2798 (2000)

103 M. A. Lisa et al (E895 Collaboration), Phys. Lett. B 496: 1 (2000)

104 U. A. Wienemann and U. Heinz, Phys. Rep 319: 145 (1999)

105 C. Y. Wong, Introduction to High-Energy Heavy-Ion Collisions (Singapore: World Scientific, 1994), Chap. 17

106 R. M. Weiner, Phys. Rep 327: 249 (2000)

107 M. Herrmann and G. F. Bertsch, Phys. Rev. C 51: 328 (1995)

108 H. J. Yin, J. Yang, W. N. Zhang, and L. L. Yu, Phys. Rev. C 86: 024914 (2012) 